\title{
Uric acid-lowering and renoprotective effects of topiroxostat, a selective xanthine oxidoreductase inhibitor, in patients with diabetic nephropathy and hyperuricemia: a randomized, double-blind, placebo-controlled, parallel-group study (UPWARD study)
}

\author{
Takashi Wada $^{1} \cdot$ Tatsuo Hosoya $^{2} \cdot$ Daisuke Honda $^{3}$ (D) $\cdot$ Ryusuke Sakamoto $^{4} \cdot$ Kazutaka Narita $^{3} \cdot$ Tomomitsu Sasaki $^{5}$. \\ Daisuke Okui ${ }^{5} \cdot K_{\text {Kenjiro Kimura }}{ }^{6}$
}

Received: 12 September 2017 / Accepted: 4 January 2018 / Published online: 25 January 2018

(c) The Author(s) 2018. This article is an open access publication

\begin{abstract}
Background Hyperuricemia is supposed to be an independent risk factor for kidney dysfunction in diabetic patients. We attempted to examine the uric acid-lowering effect and the renoprotective effect of topiroxostat, a selective xanthine oxidoreductase inhibitor, in patients with diabetic nephropathy and hyperuricemia in this pilot study.

Methods The study design was randomized, double-blind, placebo-controlled, parallel-group study. A total of 65 patients with hyperuricemia and diabetic nephropathy with microalbuminuria were enrolled and assigned to either the topiroxostat group or the placebo group. Topiroxostat (stepwise dosing from 40 to $160 \mathrm{mg}$ /day) or matching placebo was administered BID for 28 weeks. The primary endpoint was a change in the urinary albumin-to-creatinine ratio in the first-morning-void urine sample. Secondary endpoints were changes in the estimated glomerular filtration rate and the serum uric acid level. Results At 28 weeks, there was no significant difference in the percent change from baseline in the urinary albumin-tocreatinine ratio between the two groups (topiroxostat: 0 vs. placebo: $17 \%, p=0.3206$ ), but the changes in the estimated glomerular filtration rate $\left(-0.2 \mathrm{vs} .-4.0 \mathrm{~mL} / \mathrm{min} / 1.73 \mathrm{~m}^{2}, p=0.0303\right)$ and the serum uric acid level $(-2.94 \mathrm{vs} .-0.20 \mathrm{mg} /$ $\mathrm{dL}, p<0.0001$ ) were significantly different between the topiroxostat and placebo groups. Gouty arthritis occurred in 1 patient in the placebo group and no patients in the topiroxostat group.

Conclusion These findings support that diabetic nephropathy combined with hyperuricemia may be associated with kidney dysfunctions. Topiroxostat provides strict control of the serum uric acid level preventing decline of eGFR in these patients.
\end{abstract}

Keywords Hyperuricemia $\cdot$ Diabetic nephropathy $\cdot$ Albuminuria $\cdot$ Glomerular filtration rate $\cdot$ Xanthine oxidoreductase inhibitor $\cdot$ Topiroxostat $\cdot$ FYX-051

Takashi Wada and Tatsuo Hosoya contributed equally.

Daisuke Honda

d_honda@skk-net.com

1 Department of Nephrology and Laboratory Medicine, Faculty of Medicine, Institute of Medical, Pharmaceutical and Health Sciences, Kanazawa University, Kanazawa, Ishikawa, Japan

2 Department of Pathophysiology and Therapy in Chronic Kidney Disease, Jikei University School of Medicine, Tokyo, Japan
3 Drug Development Center, Sanwa Kagaku Kenkyusho Co., Ltd., Nagoya, Aichi, Japan

4 R\&D Strategy Center, Sanwa Kagaku Kenkyusho Co., Ltd., Nagoya, Aichi, Japan

5 Medical R\&D Division, Fuji Yakuhin Co., Ltd., Saitama, Japan

6 JCHO Tokyo Takanawa Hospital, Tokyo, Japan 


\section{Introduction}

Diabetic mellitus is a major risk factor of chronic kidney disease in many countries around the world [1]. Moreover, diabetic kidney disease is a leading cause of end-stage renal disease [2]. It has been shown that combination therapy with different interventions (e.g. glycemic control, amelioration of lipid metabolism, and control of hypertension, especially blockage of the renin-angiotensin system) can reduce the risk for the progression of kidney dysfunction [3]. However, drug development for the prevention of diabetic nephropathy and its progression to end-stage renal disease is still not successful. It, therefore, remains an important medical challenge to find effective strategies against diabetic kidney disease.

Meanwhile, on the basis of the well-known relationship between serum uric acid (SUA) level and renal function deterioration [4, 5], hyperuricemia is an independent risk factor [6] that impairs each of renal functions, i.e. glomerular filtration rate and albuminuria. It is pointed out that xanthine oxidoreductase (XOR), which converts hypoxanthine to xanthine, and xanthine to uric acid (UA), generates reactive oxygen species during the process of purine metabolism. Therefore, not UA excretion but UA generation via XOR is expected to be the therapeutic target for diseases involving inflammation and oxidative stress, e.g. vascular injury and renal dysfunction [7, 8]. Topiroxostat, a selective xanthine oxidoreductase inhibitor, is used for the management of hyperuricemia in patients with or without gout in Japan [9-11]. Topiroxostat not only effectively reduced SUA levels, but also had a urinary albumin-to-creatinine ratio (UACR)-lowering effect in hyperuricemic stage 3 chronic kidney disease patients [12]. Also, topiroxostat reduced the urinary albumin excretion remarkably in $\mathrm{db} / \mathrm{db}$ mice compared with febuxostat, another xanthine oxidoreductase inhibitor. It is suggested that this difference is associated with topiroxostat's potent inhibition of the plasma xanthine oxidoreductase activity [13]. Based on these facts, we conducted this pilot study named "UPWARD" to investigate the UA-lowering effect and exploratory renoprotective effect of topiroxostat in patients with early diabetic nephropathy and hyperuricemia and/or gout.

\section{Methods}

\section{Study design}

This study was carried out as phase 2 a study. The study design was a 28 -week, multicenter, randomized, doubleblind, placebo-controlled, parallel-group study. We conducted the study at 12 hospitals in Japan.

\section{Inclusion and exclusion criteria}

Patients who met all of the inclusion criteria and did not meet any exclusion criteria were enrolled in the study. The inclusion criteria were as follows: age 20-75 years; having diabetes receiving drug treatment; on constant diet and/or exercise therapy for more than 8 weeks before the start of the pre-observation period; having clinically or pathologically diagnosed diabetic kidney disease; diagnosed with gout or hyperuricemia; three measurements of first-morning-void UACR in the observation period (Visit 1 or 2), more than two of which were $\geq 45$ and $<300 \mathrm{mg} / \mathrm{gCr}$; estimated glomerular filtration ratio (eGFR) was $\geq 30 \mathrm{~mL} / \mathrm{min} / 1.73 \mathrm{~m}^{2}$ at Visit 1; outpatient (having no plan of hospital admission); and patients who can provide voluntary informed consent. The exclusion criteria were as follows: having renal diseases other than diabetic nephropathy (excluding nephrosclerosis); having received steroid preparations and immunosuppressants for the purpose of treating nephropathy within the past 10 years; having other systemic diseases than diabetes that induce proteinuria, e.g. collagen disease, vasculitis, amyloidosis, etc.; having a serum creatinine level that changed by $0.5 \mathrm{mg} / \mathrm{dL}$ or more at Visit 1 and 2; SUA level was $10.0 \mathrm{mg} /$ $\mathrm{dL}$ or more at Visit 1; having a history of nephrectomy or renal transplantation; acute renal injury within 24 weeks before the start of the pre-observation period; hepatic dysfunction, e.g. aspartate aminotransferase (AST) or alanine aminotransferase (ALT) 100 $\geq \mathrm{IU} / \mathrm{L}$; serious heart disease; serious hematologic disease; cancer; uncontrolled hypertension; uncontrolled diabetes; uncontrolled dyslipidemia; pregnancy; breastfeeding; onset of gouty arthritis at the start of the observation period; using UA-lowering agents, agents potentially affecting the SUA level and/or agents that could potentially cause adverse drug interactions with the study drug for at least 2 weeks before Visit 1 ; judged to be clinically inappropriate by the investigators or sub-investigators.

\section{Randomization, intervention, and blinding}

The study plan is shown in Fig. 1. In the Pre-observation period, we screened and enrolled eligible subjects. The patients were assigned (2:1) to either the topiroxostat group or the placebo group at the central registration center. The allocation factors were as follows: (1) first-morning-void UACR is $\geq 170 \mathrm{mg} / \mathrm{gCr}$ or not; (2) using a renin-angiotensin system inhibitor or not. Topiroxostat or matching placebo was administered BID at an initial dose of $40 \mathrm{mg}$ /day for 4 weeks, followed by stepwise increase of the dose to $160 \mathrm{mg} /$ day as shown in Fig. 1. Concomitant therapeutic agents for diabetes, hypertension (including RAS blockers), or dyslipidemia had to remain as constant as possible during the entire study period. SUA levels measured after 
Fig. 1 Study scheme

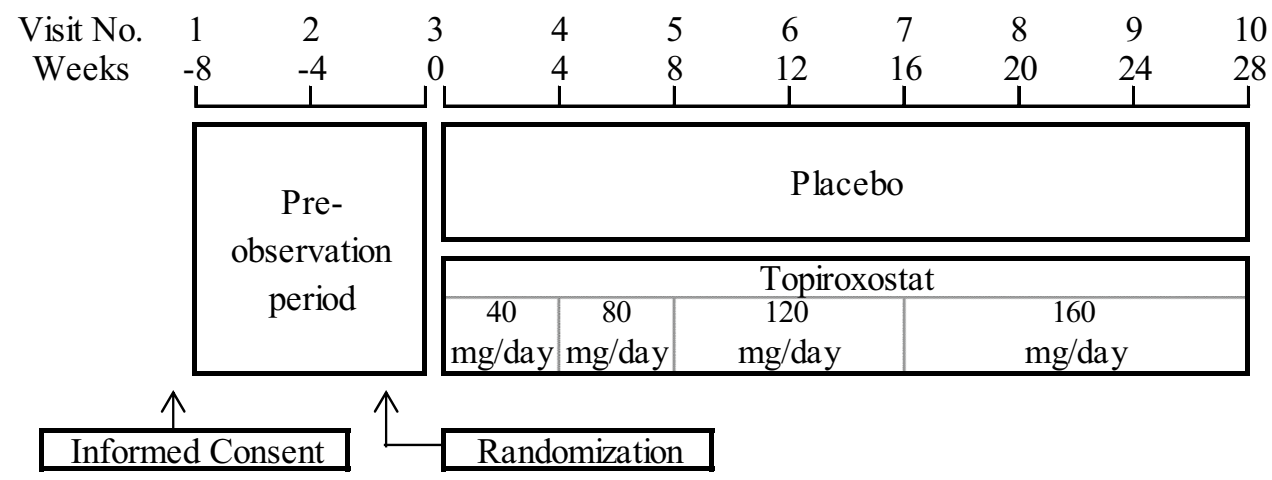

administration of the study drug in each patient were concealed from all of the investigators and the patients throughout the study period to maintain blinding. Randomization was performed independently by an outsourcing agency (A2 Healthcare Corp., Japan).

\section{Efficacy endpoints}

The primary efficacy endpoint was change in UACR in the first-morning-void urine, measured twice at each visit, and the calculated geometric mean, from baseline to the last visit. The secondary efficacy endpoints were changes in UACR in spot urine, eGFR and SUA levels.

\section{Safety evaluations}

To assess the safety and tolerability of study drug treatment, vital signs, 12-lead electrocardiography, and clinical laboratory values were observed at each visit during the study. The investigators assessed any adverse events (AEs) and their severity. AEs judged to be related to the investigational drug were defined as adverse drug reactions (ADRs). AEs and ADRs were classified according to the system organ class and preferred term (MedDRA version 18.0; Japanese Maintenance Organization, Tokyo, Japan). The incidences of AEs and ADRs that were serious or led to discontinuation of the study were summarized by the number of patients and percentage in each study group.

\section{Statistical analyses}

The baseline characteristics were summarized by study group using appropriate descriptive statistics. All efficacy analyses at the endpoints were conducted under the full analysis set principle. For assessment of the UACR change, we performed logarithmic transformation of the UACR value. Adjusted average value and $95 \%$ confidence interval on both sides were estimated, and the obtained results are shown by inverse logarithmic transformation. We compared the two groups for change from baseline at the last observation with the analysis of covariance (ANCOVA) model. To analyze the UACR in the firstmorning-void urine, the baseline UACR, and the use of angiotensin II receptor blockers or angiotensin-converting enzyme inhibitors (RAS blockers) or not were selected as covariates. To assess UACR in spot urine, SUA, and eGFR, we compared the changes from baseline at the last observation with the ANCOVA model, and compared the results between the two groups; the covariates were each baseline value. If the data at the time of the last visit ( 28 weeks) were missing, it was compensated by the LOCF method.

Safety analyses were performed on the safety population, consisting of all randomized patients who received at least one dose of the study drug and who had no critical GCP violations. Fisher's exact test was used for comparison of the incidences between study groups.

To determine the number of patients, we referred to a clinical study of topiroxostat for hyperuricemic stage 3 chronic kidney disease [12]. In this study, at 22 weeks, the mean change from baseline after logarithmic conversion of UACR was -0.1772 with a standard deviation of 0.2969 in the topiroxostat group. Assuming that topiroxostat's effect on UACR did not diminish after 28 weeks of administration, it was found that more than 32 patients are needed to compare with baseline data with $\alpha=0.05$ (two-sided significance level) and $1-\beta=0.9$. Considering a possible dropout of some patients, we set the required number of patients in the topiroxostat group at 40 . To obtain a natural course of target patients in this study as a comparison, we set the number of patients in the placebo group at 20 . Since the main evaluation subject was the last observation day, we did not consider statistical multiplicity for the evaluation of each visit. All statistical analyses of efficacy and safety were performed independently by an outsourcing agency (EPS Corp., Japan) with SAS software, version 9.3 or higher (SAS Institute). 


\section{Results}

\section{Patient flow and background}

A total of 141 patients were enrolled, and 76 patients were excluded. The main reasons for withdrawal were UACR outside the target range $(n=56)$ and other unacceptable laboratory values $(n=13)$. Of the remaining 65 patients who were randomized, all patients received topiroxostat or matching placebo. Two patients in the topiroxostat group and 4 patients in the placebo group failed to complete the study, but no patients were excluded from the full analysis set (Fig. 2).

The baseline characteristics of the randomized population are shown in Table 1. The baseline UACR was $141.5 \mathrm{mg} / \mathrm{gCr}$ (95\% CI $106.30-188.37 \mathrm{mg} / \mathrm{gCr}$ ) in the placebo group and $114.5 \mathrm{mg} / \mathrm{gCr}$ (95\% CI $92.26-142.10 \mathrm{mg} /$ $\mathrm{gCr}$ ) in the topiroxostat group, without significant differences between the groups (two-sample $t$ test with logarithmic transformation values; $p=0.2416$ ). The backgrounds of the two groups were similar, except for total cholesterol and low-density lipoprotein cholesterol (LDL), which were significantly lower in the placebo group.
Efficacy

\section{UACR in first morning void urine}

The time course of the geometric mean of UACR in firstmorning-void urine is shown in Fig. 3a. The percent increase in the UACR level in the first-morning-void urine from baseline to the last visit was $17 \%$ (95\% CI - 9 to $52 \%$ ) in the placebo group $(n=22)$ and $0 \%(95 \% \mathrm{CI}-17$ to $20 \%)$ in the topiroxostat group $(n=43)$ with a between-group difference of $-15 \%$ (95\% CI -38 to $17 \%)(p=0.3206)$ (Fig. 4a). While UACR in the first-morning-void urine was upward in the placebo group, it was unchanged in the topiroxostat group. However, a statistically significant difference was not observed.

The numbers of patients who had normalized UACR $(<30 \mathrm{mg} / \mathrm{gCr})$ at the last observation was $0(0 \%)$ in the placebo group and $2(4.7 \%)$ in the topiroxostat group.

\section{UACR in spot urine}

The time course of the geometric mean of UACR in spot urine is shown in Fig. 3b. The percent increase in UACR in the spot urine level from baseline to the last visit was $28 \%(95 \% \mathrm{CI}-2$ to $69 \%)$ in the placebo group $(n=22)$ and $-2 \%(95 \% \mathrm{CI}-20$ to $19 \%)$ in the topiroxostat group $(n=43)$ with a between-group difference of $-24 \%(95 \%$
Fig. 2 Flow diagram for two groups of the study

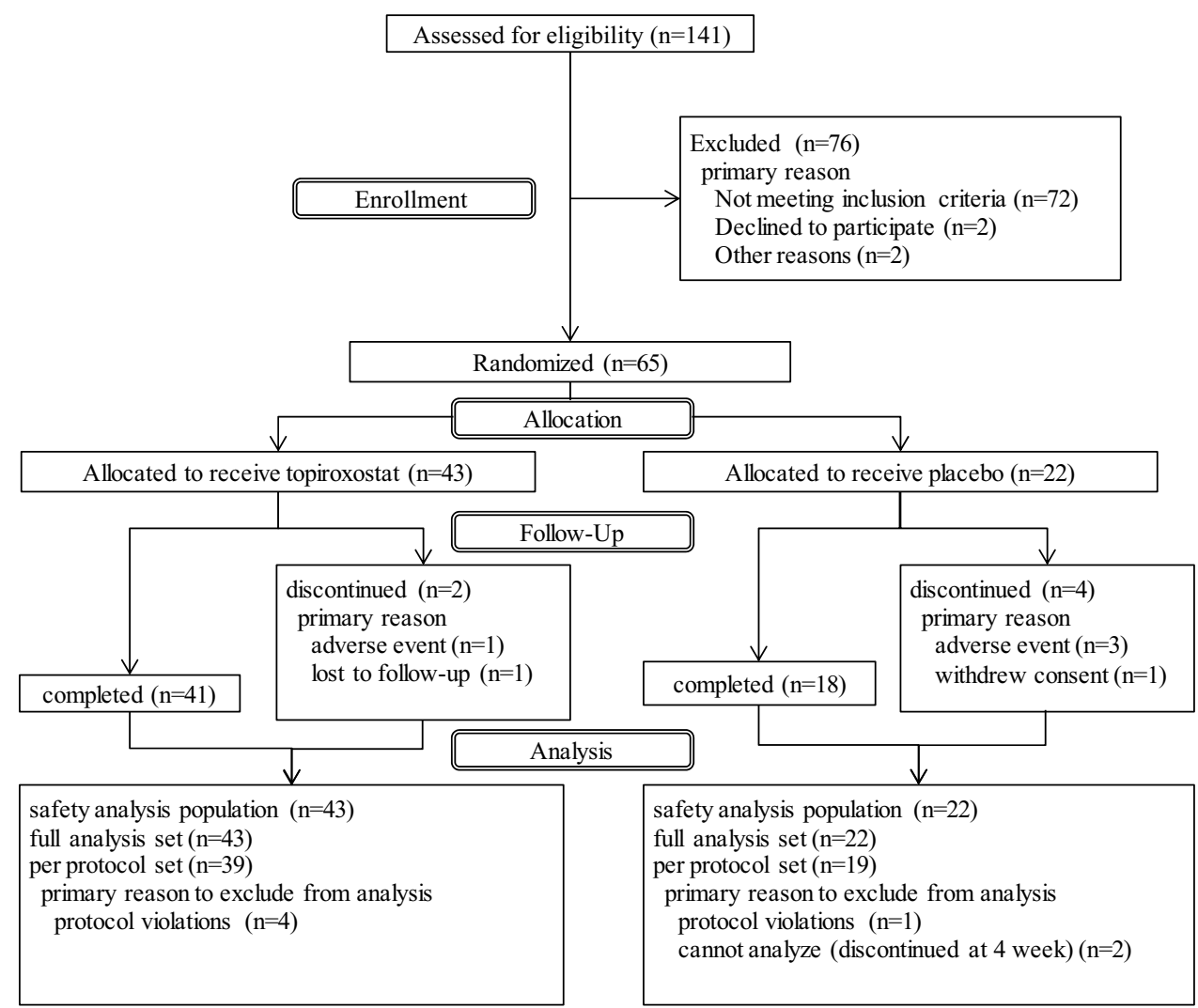


Table 1 Baseline characteristics of randomized population at visit 2

\begin{tabular}{llll}
\hline Variable & Placebo group $(n=22)$ & Topiroxostat group $(n=43)$ & $p$ value \\
\hline Age (years) & $63.0 \pm 8.7$ & $60.5 \pm 10.1$ & $0.3351^{\mathrm{b}}$ \\
Male, $n(\%)$ & $20(90.9)$ & $37(86.0)$ & $0.7063^{\mathrm{c}}$ \\
Weight $(\mathrm{kg})$ & $73.77 \pm 11.10$ & $74.65 \pm 11.52$ & $0.7676^{\mathrm{b}}$ \\
BMI $\left(\mathrm{kg} / \mathrm{m}^{2}\right)$ & $26.08 \pm 2.80$ & $27.17 \pm 4.23$ & $0.2816^{\mathrm{b}}$ \\
Duration of DM (years) & $15.49 \pm 8.99$ & $17.84 \pm 10.25$ & $0.3733^{\mathrm{b}}$ \\
Type 2 DM, $n(\%)$ & $21(95.5)$ & $42(97.7)$ & $1.0000^{\mathrm{c}}$ \\
UACR in first morning void urine & $141.5[106.30,188.37]$ & $114.5[92.26,142.10]$ & $0.2416^{\mathrm{d}}$ \\
(mg/gCr) & & & \\
UACR in spot urine (mg/gCr) & & \\
eGFR (mL/min/1.73 m $\left.{ }^{\mathrm{a}}\right)$ & $234.74[170.90,322.45]$ & $221.49[169.17,289.99]$ & $0.7892^{\mathrm{d}}$ \\
SUA (mg/dL) & $68.3 \pm 21.7$ & $66.3 \pm 17.6$ & $0.6909^{\mathrm{b}}$ \\
HbA1c $(\%)$ & $7.03 \pm 1.13$ & $7.25 \pm 1.19$ & $0.4818^{\mathrm{b}}$ \\
SBP (mmHg) & $7.01 \pm 1.11$ & $7.30 \pm 1.28$ & $0.3729^{\mathrm{b}}$ \\
DBP (mmHg) & $130.4 \pm 10.2$ & $130.3 \pm 14.3$ & $0.9645^{\mathrm{b}}$ \\
Total cholesterol (mg/dL) & $74.5 \pm 11.3$ & $77.5 \pm 10.3$ & $0.2974^{\mathrm{b}}$ \\
LDL (mg/dL) & $162.0 \pm 29.8$ & $186.7 \pm 31.7$ & $0.0036^{\mathrm{b}}$ \\
HDL (mg/dL) & $100.3 \pm 27.4$ & $112.9 \pm 25.4$ & $0.0703^{\mathrm{b}}$ \\
RAS blockers, $n(\%)$ & $45.8 \pm 9.5$ & $50.0 \pm 13.6$ & $0.2001^{\mathrm{b}}$ \\
Complications, $n(\%)$ & $18(81.8)$ & $36(83.7)$ & $1.0000^{\mathrm{c}}$ \\
Diabetic retinopathy & & & \\
Diabetic neuropathy & $12(54.5)$ & $23(53.5)$ & $1.0000^{\mathrm{c}}$ \\
Hypertension & $8(36.4)$ & $21(48.8)$ & $0.4319^{\mathrm{c}}$ \\
Dyslipidemia & $19(86.4)$ & $40(93.0)$ & $0.3985^{\mathrm{c}}$ \\
\hline
\end{tabular}

Mean \pm SD or $n(\%)$

$B M I$ Body mass index, $D M$ diabetes mellitus, $S B P$ systolic blood pressure, $D B P$ diastolic blood pressure, $H b A 1 c$ glycosylated hemoglobin, $U A C R$ urinary albumin-to-creatinine ratio, SUA serum uric acid, $e G F R$ estimated glomerular filtration rate, $L D L$ low-density lipoprotein cholesterol, $H D L$ high-density lipoprotein cholesterol, $R A S$ blockers, use of angiotensin II receptor blockers or angiotensin-converting enzyme inhibitors

${ }^{a}$ UACRs were listed a geometric mean and $95 \%$ confidence interval

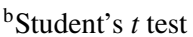

${ }^{\mathrm{c}}$ Fisher's exact test

${ }^{\mathrm{d}}$ Student's $t$ test after logarithmic transformation
CI -54 to $7 \%)(p=0.1103)$ (Fig. 4b). Changes in UACR in spot urine were similar to those in the first-morningvoid urine.

\section{eGFR}

The time course of mean eGFR is shown in Fig. 3c. The mean change in eGFR level from baseline to the last visit was -4.0 (95\% CI -6.7 to -1.2$)$ in the placebo group $(n=22)$ and $-0.2(95 \%$ CI -2.2 to 1.7$)$ in the topiroxostat group $(n=43)$ with a between-group difference of 3.7 (95\% CI 0.4 to 7.1$)(p=0.0303)$ (Fig. 4c). The eGFR declined in the placebo group, but was unchanged in the topiroxostat group. Moreover, the difference was statistically significant.

\section{SUA}

The time course of mean SUA is shown in Fig. 3d. The mean change in SUA from baseline to the last visit was -0.20 (95\% CI -0.58 to 0.18 ) in the placebo group $(n=22)$ and $-2.94(95 \% \mathrm{CI}-3.21$ to -2.66$)$ in the topiroxostat group $(n=43)$ with a between-group difference of $-2.74(95 \% \mathrm{CI}-3.20$ to -2.27$)(p<0.0001)$ (Fig. 4d). SUA did not change in the placebo group, but decreased in the topiroxostat group. The difference was statistically significant.

The numbers of patients who had normalized SUA ( $>6.0 \mathrm{mg} / \mathrm{dL}$ at baseline, and $\leq 6.0 \mathrm{mg} / \mathrm{dL}$ at the last observation) were $33(91.7 \%)$ in the topiroxostat group and $0(0 \%)$ in the placebo group. 


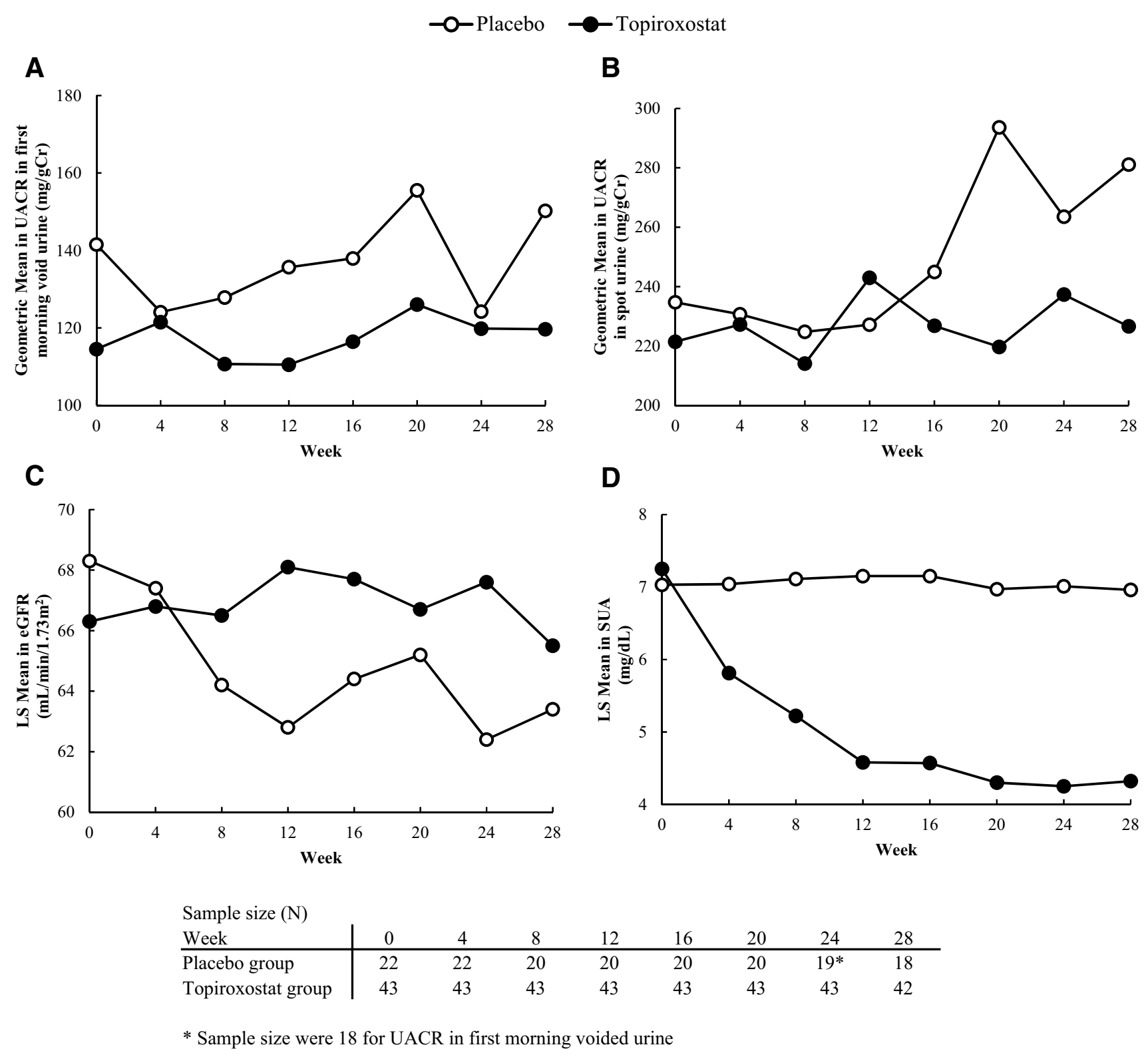

Fig. 3 Time course of mean value. a UACR in first-morning-void urine (geometric mean). b UACR in spot urine (geometric mean). c eGFR. d SUA

\section{Safety}

\section{Adverse events}

Adverse events occurred in 11 patients (50.0\%) in the placebo group and 32 patients $(74.4 \%)$ in the topiroxostat group. No between-group difference was observed for the incidence of adverse events (Fisher's exact test; $p=0.0585$ ). No deaths were observed in either group during the study period. Serious adverse events were observed in 3 patients ( 3 cases) in the topiroxostat group and 2 patients $(3$ cases) in the placebo group. The breakdown is as follows: "diabetic retinopathy $(n=2)$ " and "colon cancer $(n=1)$ " in the topiroxostat group, and "lumbar spinal stenosis $(n=1)$ ", "intervertebral disc disorder $(n=1)$ ", and "hepatocellular carcinoma $(n=1)$ " in the placebo group. A causal relationship of serious adverse events with the investigational drug was denied.

Four patients in the placebo group and 1 patient in the topiroxostat group withdrew from this study due to adverse events. The breakdown is as follows: "intervertebral disc disorder and lumbar spinal stenosis $(n=1)$ ", "hyperglycaemia $(n=2)$ ", and "hepatocellular carcinoma $(n=1)$ " 
Fig. 4 Change of last observation value from baseline. a UACR in first morning void urine (percent change and 95\% confidence interval). b UACR in spot urine (percent change and $95 \%$ confidence intervaln). c eGFR (mean change and 95\% confidence interval). d SUA (mean change and 95\% confidence interval)
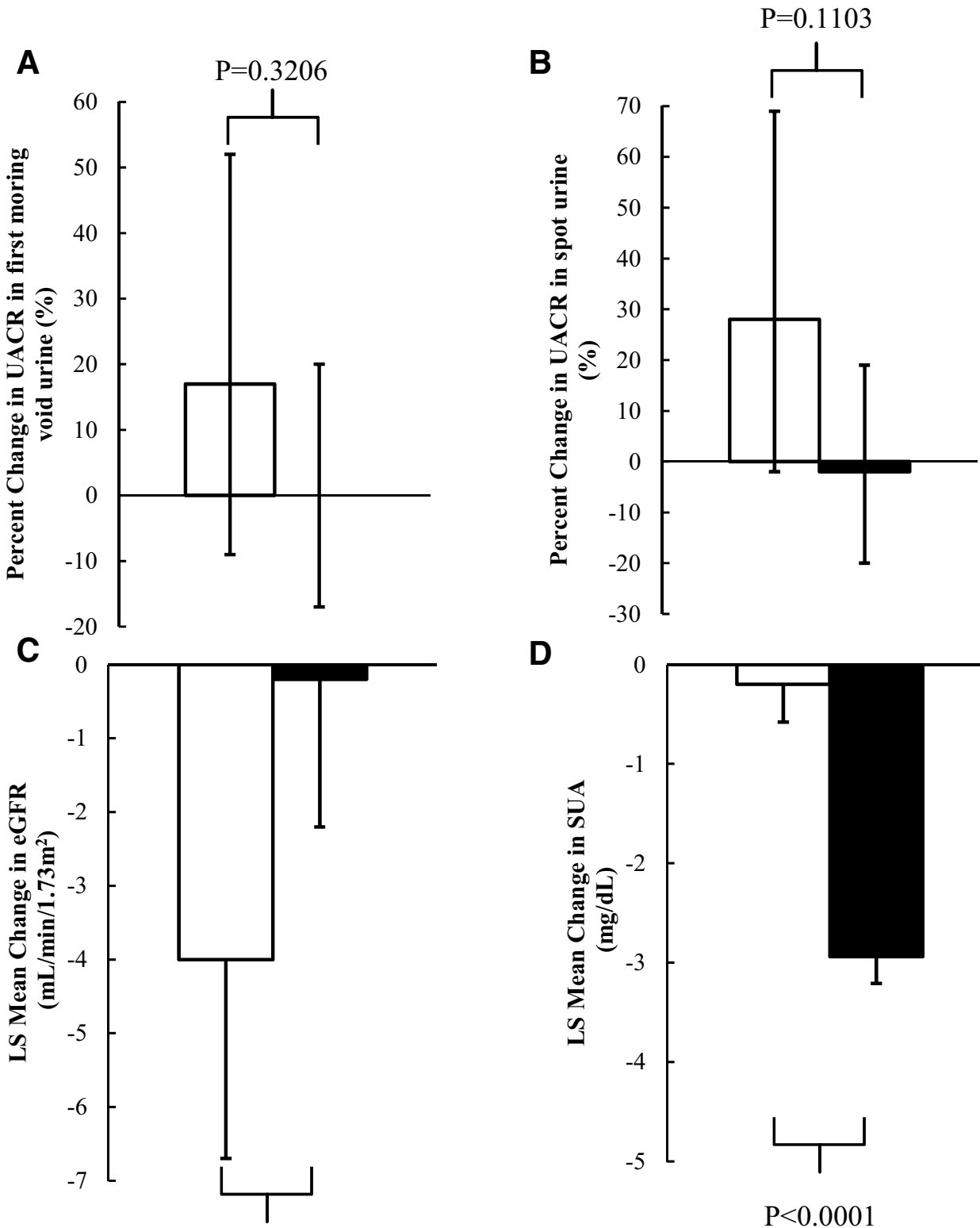

D

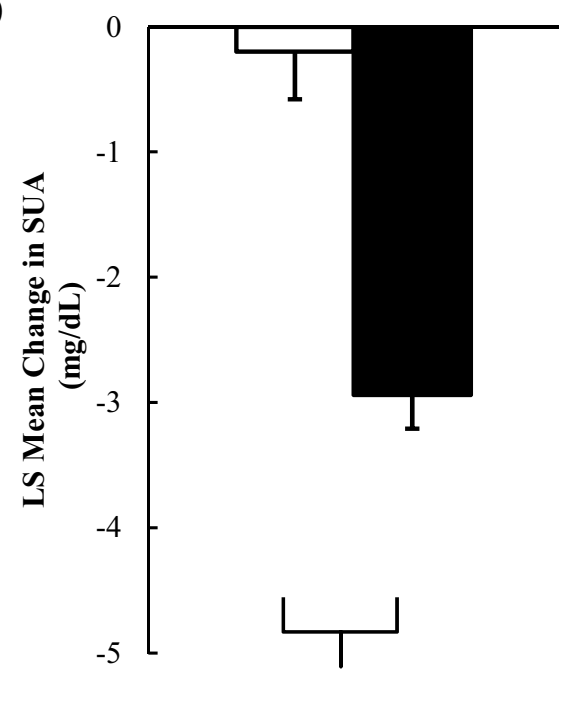

$\mathrm{P}<0.0001$

$\mathrm{P}=0.0303$

Sample size $(\mathrm{N})$

Placebo group Topiroxostat group 22

in the placebo group, and "colon cancer $(n=1)$ " in the topiroxostat group.

"Arthritis $(n=1)$ " and "gouty arthritis $(n=1)$ " were reported as adverse drug reactions in the placebo group, and "alanine aminotransferase increase $(n=1)$ " and "aspartate aminotransferase increase $(n=1)$ " were reported as adverse drug reactions in the topiroxostat group (Table 2).

\section{Control of complications}

The time course of the mean values of glycosylated hemoglobin (HbA1c), systolic blood pressure (SBP), diastolic blood pressure (DBP), LDL, and high-density lipoprotein cholesterol (HDL) is shown in Fig. 5. The treatment of diabetes, dyslipidemia, and hypertension in each study group remained the same and the diseases were well-controlled during the entire study period. There were no significant 
Table 2 Summary of adverse events

\begin{tabular}{lll}
\hline Adverse events $(\mathrm{PT})$ & \multicolumn{2}{l}{ Number $(\%)$ of patients } \\
\cline { 3 - 3 } & Placebo $(N=22)$ & $\begin{array}{l}\text { Topiroxostat } \\
(N=43)\end{array}$ \\
\hline Any AEs & $11(50.0)$ & $32(74.4)$ \\
Serious AEs & $2(9.1)$ & $3(7.0)$ \\
AEs caused withdrawal & $4(18.2)$ & $1(2.3)$ \\
AEs occurring in $\geq 5 \%$ of patients in either group & & $3(7.0)$ \\
Diabetic retinopathy & & $3(7.0)$ \\
Seasonal allergy & $1(4.5)$ & $9(20.9)$ \\
Nasopharyngitis & $3(13.6)$ & $1(2.3)$ \\
Adverse drug reactions & & $1(2.3)$ \\
Alanine aminotransferase increase & & \\
Aspartate aminotransferase increase & & \\
Arthritis & $1(4.5)$ & $1(4.5)$ \\
Gouty arthritis & & \\
\hline
\end{tabular}

$A E$ adverse event, $P T$ preferred term specified by MedDRA version 18.0 differences in SBP, DBP, LDL, or HDL. However, the mean change in HbA1c level from baseline to the last visit was 0.48 (95\% CI 0.19 to 0.77$)$ in the placebo group $(n=21)$ and 0.08 (95\% CI -0.12 to 0.29$)$ in the topiroxostat group $(n=43)$ with a between-group difference of -0.40 (95\% CI -0.76 to -0.04$)(p=0.0303)$ analyzed by the ANCOVA model; the covariate was baseline HbA1c. To examine whether increase in $\mathrm{HbAlc}$ affects decrease in eGFR, we performed simple linear regression analysis. As a result, the regression equation: eGFR change $=-1.34789$ $-0.66108 \times \mathrm{HbA} 1 \mathrm{c}$ change, and coefficient of determination: 0.0048 , were obtained. Therefore, increase in $\mathrm{HbA} 1 \mathrm{c}$ did not affect decrease in eGFR.

\section{Discussion}

This is the first double-blind study involving topiroxostat for patients with diabetic nephropathy with microalbuminuria. We showed that a stepwise increase of topiroxostat to a dose of $160 \mathrm{mg} /$ day effectively reduced the SUA level in patients with diabetic nephropathy combined with hyperuricemia. Adverse drug reactions observed in this study were only mild elevations of AST and ALT, which had been reported in previous studies [9] in one patient. Furthermore, in the topiroxostat group, no patients had onset of gout attacks which were often observed under the strict control of SUA.

Concerning albuminuria, UACR levels tended to increase in the placebo group, while they did not tend to increase in the topiroxostat group. However, no statistically significant difference was observed in the change in UACR between the topiroxostat group and the placebo group. We referred to a clinical study of topiroxostat in patients with hyperuricemic stage 3 chronic kidney disease
[12]. In this study, the standard deviation of change in UACR in the topiroxostat group was 0.297. Meanwhile, in the UPWARD study, the standard deviation of change in UACR in the topiroxostat group was 0.557 . These results might support the heterogeneousness of the inpatients with albuminuria examined in this study. Several studies suggest that hyperuricemia may be a factor of the progress of albuminuria in diabetic nephropathy $[14,15]$ and that XOR inhibitors may reduce albuminuria [16-18]. On the other hand, there were few studies that showed uricosuric agents without XOR inhibitory potency may reduce albuminuria. The relationship between XOR activity and albuminuria should be examined in the future.

As for eGFR, we found a significant difference in eGFR changes between the topiroxostat group and the placebo group. Interestingly, although enrollment of eligible patients, randomization, and blinding were conducted properly, the eGFR in the placebo group declined $6 \%$ within half a year. It was reported that the rate of eGFR decline in diabetic patients with microalbuminuria is about $1.0-2.0 \%$ per year $[19,20]$. Hence eGFR of patients in this study seemed to decline rapidly. Diabetes, hypertension [21-26], and dyslipidemia [27] are well known as risk factors for renal function deterioration. Patients in this study had these multiple risk factors, however, these patients' complications were well controlled, and their biomarkers did not fluctuate sharply. In particular, almost all patients received RAS blockers constantly without changing dosage during the study period. The only significant difference of markers between the placebo group patients and the topiroxostat group patients was SUA level.

Recent studies have shown that elevated SUA levels may be associated with the faster progression of diabetic kidney disease [28-30]. The present data indicate that these 
Fig. 5 Time course of mean value in complication biomarkers. a HbA1c. b SBP and DBP. c LDL and HDL
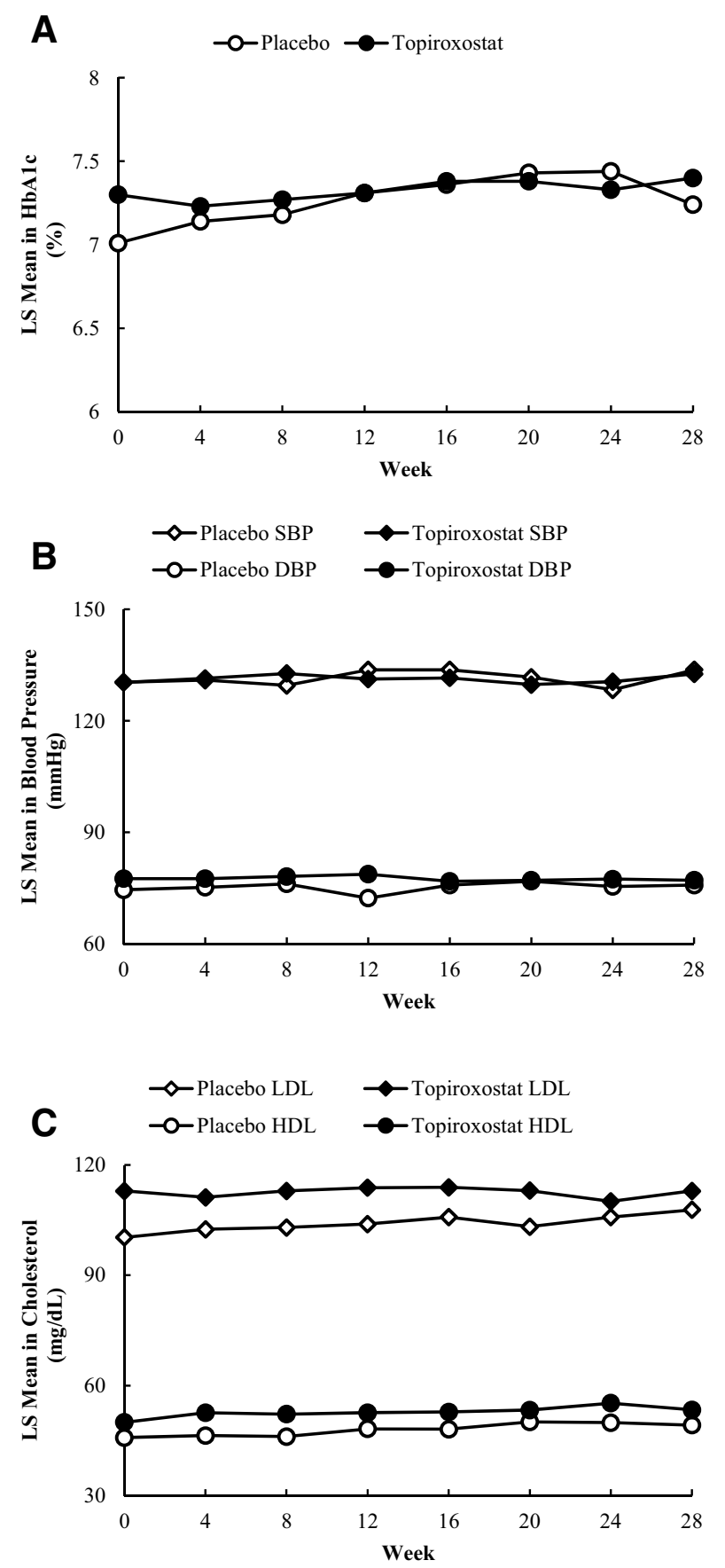

\begin{tabular}{l|cccccccc} 
Sample size (N) & 0 & 4 & 8 & 12 & 16 & 20 & 24 & 28 \\
Week & 22 & 22 & 20 & 20 & 20 & 20 & 19 & 18 \\
\hline Placebo group & 43 & 43 & 43 & 43 & 43 & 43 & 43 & 42
\end{tabular}

past findings may be applicable to early stage diabetic nephropathy.
Although UA-lowering therapy for preventing and slowing kidney disease progression has not been established, the result of this study suggests that appropriate 
management of hyperuricemia at an early stage of diabetic kidney disease is likely to be important for renal protection.

Furthermore, XOR activity is connected with endothelial dysfunction via inflammation and oxidative stress [31, 32], and XOR in diabetic patients is found to be activated [33, 34], as previously reported. Since XOR inhibitors reduce the production of reactive oxygen species $[35,36]$ in addition to lowering of the UA level, topiroxostat has the possibility to provide renal protection through XOR inhibition.

There are several limitations to this study. The first point is that this study is just a pilot study involving a small number of patients and only half a year of observation. Therefore, robustness and reproducibility may be insufficient. Large-scale and long-term observation may be necessary to confirm whether changes in eGFR obtained as a result of this study are general facts. In addition, we could not find a statistically significant difference in UACR. The second point is that patients included in this study are early stage diabetic nephropathy patients with microalbuminuria. Similar intervention studies for patients with overt albuminuria with UACR $>300$ or those with even lower renal function, e.g. GFR of 30 or less, are considered as a future task in progression of nephropathy. Recently, a study involving hyperuricemic patients with diabetic nephropathy and overt proteinuria was reported [37, 38]. In this report, topiroxostat had a steady anti-albuminuric effect in patients with type 2 diabetes and overt proteinuria who were at high risk of progressive diabetic nephropathy. This investigation might be an advance in the evaluation of the potential of topiroxostat to reduce albuminuria. The third point is that this study targeted only patients diagnosed with gout or hyperuricemia. Even if topiroxostat has a renal protective effect in case of diabetic nephropathy, it is unknown whether such effect is completely independent of reduced uric acid level. We should confirm this with a study in diabetic nephropathy patients having normal uric acid level.

In conclusion, the results of this study demonstrated that treatment with topiroxostat effectively reduced the SUA levels with good tolerability and safety in hyperuricemic patients with early diabetic nephropathy. Although further clinical studies are necessary to validate this potential, topiroxostat appears to suppress the decline in renal function in patients with hyperuricemia combined with diabetic nephropathy.

Acknowledgements We are grateful to the investigators, local study coordinators and the patients for their valuable contributions to this study; Takahito Jomori, managing director of Sanwa Kagaku Kenkyusho Co., Ltd. (SKK), for carefully proofreading the manuscript.

\section{Compliance with ethical standards}

Ethical standards The study protocol and informed consent document were reviewed and approved by health authority in Japan (Pharmaceuticals and Medical Devices Agency) and the investigation review board of each medical institution. Informed consent was obtained from all individual participants included in the study. The tests were conducted in compliance with the 1964 Helsinki Declaration and its later amendments or comparable ethical standards, Good Clinical Practice guidelines, and other regulatory requirements to be applied. The information of this study was registered with Clinical Trials.gov (ClinicalTrials.gov number: NCT02327754).

Conflict of interest SKK and Fuji Yakuhin Co., Ltd. (Fuji) sponsored this study. TW, TH, KK were advisors to SKK and Fuji regarding this study and received consultant fees. TH's laboratory has received research fund from SKK and Fuji. DH, RS, KN were employees of SKK. TS, DO were employees of Fuji.

Open Access This article is distributed under the terms of the Creative Commons Attribution 4.0 International License (http://creativecommons.org/licenses/by/4.0/), which permits unrestricted use, distribution, and reproduction in any medium, provided you give appropriate credit to the original author(s) and the source, provide a link to the Creative Commons license, and indicate if changes were made.

\section{References}

1. Webster AC, Nagler EV, Morton RL, Masson P. Chronic kidney disease. Lancet. 2017;389:1238-52.

2. Tuttle KR, Bakris GL, Bilous RW et al. Diabetic kidney disease: a report from an ADA consensus conference. Diabetes Care. 2014;37:2864-83.

3. Molitch ME, Adler AI, Flyvbjerg A et al. Diabetic kidney disease-a clinical update from kidney disease: improving global outcomes (KDIGO). Kidney Int. 2015;87:20-30.

4. Obermayr RP, Temml C, Gutjahr G, Knechtelsdorfer M, Oberbauer R, Klauser-Braun R. Elevated uric acid increases the risk for kidney disease. J Am Soc Nephrol. 2008;19:2407-13.

5. Takae K, Nagata M, Hata J, et al. Serum uric acid as a risk factor for chronic kidney disease in a Japanese community. Circ J. 2016;80:1857-62.

6. Kuwabara M, Bjornstad P, Hisatome I, et al. Elevated serum uric acid level predicts rapid decline in kidney function. Am J Nephrol. 2017;45:330-37.

7. Jalal DI, Chonchol M, Chen W, Targher G. Uric acid as a target of therapy in CKD. Am J Kidney Dis. 2013;61:134-46.

8. Kushiyama A, Tanaka K, Hara S, Kawazu S. Linking uric acid metabolism to diabetic complications. World J Diabetes. 2014;5:787-95.

9. Hosoya T, Sasaki T, Hashimoto H, Sakamoto R, Ohashi T. Clinical efficacy and safety of topiroxostat in Japanese male hyperuricemic patients with or without gout: an exploratory, phase $2 \mathrm{a}$, multicentre, randomized, double-blind, placebo-controlled study. J Clin Pharm Ther. 2016;41:298-305.

10. Hosoya T, Sasaki T, Ohashi T. Clinical efficacy and safety of topiroxostat in Japanese hyperuricemic patients with or without gout: a randomized, double-blinded, controlled phase $2 \mathrm{~b}$ study. Clin Rheumatol. 2017;36:649-56.

11. Hosoya T, Ogawa Y, Hashimoto H, Ohashi T, Sakamoto R. Comparison of topiroxostat and allopurinol in Japanese hyperuricemic 
patients with or without gout: a phase 3, multicentre, randomized, double-blind, double-dummy, active-controlled, parallel-group study. J Clin Pharm Ther. 2016;41:290-7.

12. Hosoya T, Ohno I, Nomura $S$, et al. Effects of topiroxostat on the serum urate levels and urinary albumin excretion in hyperuricemic stage 3 chronic kidney disease patients with or without gout. Clin Exp Nephrol. 2014;18:876-84.

13. Nakamura T, Murase T, Nampei M, Morimoto N, Ashizawa N, Iwanaga T, Sakamoto R. Effects of topiroxostat and febuxostat on urinary albumin excretion and plasma xanthine oxidoreductase activity in db/db mice. Eur J Pharmacol. 2016;780:224-31.

14. Behradmanesh S, Horestani MK, Baradaran A, Nasri H. Association of serum uric acid with proteinuria in type 2 diabetic patients. J Res Med Sci. 2013;18:44-6.

15. Kim WJ, Kim SS, Bae MJ, et al. High-normal serum uric acid predicts the development of chronic kidney disease in patients with type 2 diabetes mellitus and preserved kidney function. J Diabetes Complications. 2014;28:130-4.

16. Momeni A, Shahidi S, Seirafian S, Taheri S, Kheiri S. Effect of allopurinol in decreasing proteinuria in type 2 diabetic patients. Iran J Kidney Dis. 2010;4:128-32.

17. Liu P, Chen Y, Wang B, et al. Allopurinol treatment improves renal function in patients with type 2 diabetes and asymptomatic hyperuricemia: 3-year randomized parallel-controlled study. Clin Endocrinol (Oxf). 2015;83:475-82.

18. Tanaka K, Nakayama M, Kanno M, et al. Renoprotective effects of febuxostat in hyperuricemic patients with chronic kidney disease: a parallel-group, randomized, controlled trial. Clin Exp Nephrol. 2015;19:1044-53.

19. Hoefield RA, Kalra PA, Baker PG, et al. The use of eGFR and ACR to predict decline in renal function in people with diabetes. Nephrol Dial Transpl. 2011;26:887-92.

20. Araki S, Haneda M, Koya D, et al. Association between urinary type IV collagen level and deterioration of renal function in type 2 diabetic patients without overt proteinuria. Diabetes Care. 2010;33:1805-10.

21. Klag MJ, Whelton PK, Randall BL, et al. Blood pressure and end-stage renal disease in men. N Engl J Med. 1996;334:13-8.

22. Klag MJ, Whelton PK, Randall BL, Neaton JD, Brancati FL, Stamler J. End-stage renal disease in African-American and white men. 16-year MRFIT findings. JAMA. 1997;277:1293-8.

23. Tozawa M, Iseki K, Iseki C, Kinjo K, Ikemiya Y, Takishita S. Blood pressure predicts risk of developing end-stage renal disease in men and women. Hypertension. 2003;41:1341-5.

24. Vupputuri S, Batuman V, Muntner P, et al. Effect of blood pressure on early decline in kidney function among hypertensive men. Hypertension. 2003;42:1144-9.

25. Yamagata K, Ishida K, Sairenchi T, et al. Risk factors for chronic kidney disease in a community-based population: a 10-year follow-up study. Kidney Int. 2006;71:159-66.
26. Reynolds K, Gu D, Muntner P, et al. A population-based, prospective study of blood pressure and risk for end-stage renal disease in China. J Am Soc Nephrol. 2007;18:1928-35.

27. Schaeffner ES, Kurth T, Curhan GC, et al. Cholesterol and the risk of renal dysfunction in apparently healthy men. J Am Soc Nephrol. 2003;14:2084-91.

28. Tanaka K, Hara S, Hattori M, et al. Role of elevated serum uric acid levels at the onset of overt nephropathy in the risk for renal function decline in patients with type 2 diabetes. J Diabetes Investig. 2015;6:98-104.

29. Bartáková V, Kuricová K, Pácal L, et al. Hyperuricemia contributes to the faster progression of diabetic kidney disease in type 2 diabetes mellitus. J Diabetes Complicat. 2016;30:1300-7.

30. Shah P, Bjornstad P, Johnson RJ. Hyperuricemia as a potential risk factor for type 2 diabetes and diabetic nephropathy. J Bras Nefrol. 2016;38:386-7.

31. George J, Carr E, Davies J, Belch JJ, Struthers A. High-dose allopurinol improves endothelial function by profoundly reducing vascular oxidative stress and not by lowering uric acid. Circulation. 2006;114:2508-16.

32. Schulz E, Gori T, Münzel T. Oxidative stress and endothelial dysfunction in hypertension. Hypertens Res. 2011;34:665-73.

33. Kuppusamy UR, Indran M, Rokiah P. Glycaemic control in relation to xanthine oxidase and antioxidant indices in Malaysian Type 2 diabetes patients. Diabet Med. 2005;22:1343-6.

34. Suriyajothi MA, Sangeetha R, Venkateswari R. Activity of xanthine oxidase in diabetics: its correlation with aging. Pharmacologyonline. 2011;2:128-33.

35. Shin JH, Chun KS, Na YG, et al. Allopurinol protects against ischemia/reperfusion-induced injury in rat urinary bladders [published online Sep 27,2015]. Oxid Med Cell Longev. https://doi. org/10.1155/2015/906787.

36. Kamijo-Ikemori A, Sugaya T, Hibi C, et al. Renoprotective effect of the xanthine oxidoreductase inhibitor topiroxostat on adenine-induced renal injury. Am J Physiol Renal Physiol. 2016;310:F1366-76.

37. Kato S, Ando M, Mizukoshi T, et al. Randomized control trial for the assessment of the anti-albuminuric effects of topiroxostat in hyperuricemic patients with diabetic nephropathy (the ETUDE study). Nagoya J Med Sci. 2016;78:135-42.

38. Mizukoshi T, Kato S, Ando M, et al. Renoprotective effects of topiroxostat for hyperuremic patients with overt diabetic nephropathy study (ETUDE Study): a prospective, randomized, multicenter clinical trial [published online Oct 9,2017]. Nephrology. https://doi.org/10.1111/nep.13177. 